\title{
THE DUAL PROCESS MODEL OF COPING WITH BEREAVEMENT: RATIONALE AND DESCRIPTION
}

\section{Margaret Stroebe, Henk Schut}

To cite this article: Margaret Stroebe, Henk Schut (1999) THE DUAL PROCESS MODEL OF COPING WITH BEREAVEMENT: RATIONALE AND DESCRIPTION, Death Studies, 23:3, 197-224, DOI: 10.1080/074811899201046

To link to this article: https://doi.org/10.1080/074811899201046

\section{Published online: 11 Nov 2010.}

Submit your article to this journal $\pi$

Џ Article views: 11847

Q View related articles $\sqsubset$

4 Citing articles: 817 View citing articles 


\section{THE DUAL PROGESS MODEL OF COPING WITH BEREAVEMENT : RATIONALE AND DESGRIPTION}

\section{MARGARET STROEBE and HENK SCHUT}

Utrecht University, The Netherlands

There are shortcomings in traditional theorizing about effective ways of coping with bereavement, most notably, with respect to the so-called "grief work hypothesis." Criticisms include imprecise definition, failure to represent dynamic processing that is characteristic of grieving, lack of empirical evidence and validation across cultures and historical periods, and a limited focus on intrapersonal processes and on health outcomes. Therefore, a revised model of coping with bereavement, the dual process model, is proposed. This model identifies two types of stressors, loss- and restoration-oriented, and a dynamic, regulatory coping process of oscillation, whereby the grieving individual at times confronts, at other times avoids, the different tasks of grieving. This model proposes that adaptive coping is composed of confrontationavoidance of loss and restoration stressors. It also argues the need for dosage of grieving, that is, the need to take respite from dealing with either of these stressors, as an integral part of adaptive coping. Empirical research to support this conceptualization is discussed, and the model's relevance to the examination of complicated grief, analysis of subgroup phenomena, as well as interpersonal coping processes, is described.

"Grieving is crucial, necessary and unavoidable for successful adaptation." (Malkinson, 1996, p. 155)

"Those who show the most evidence of working through the loss are those who ultimately have the most difficulty in resolving what has happened." (Wortman \& Silver, 1987, p. 207)

The notion that one "has to do one's grief work" is well-known in popular as well as scientific literature on bereavement, although,

We wish to thank Robert Neimeyer and three anonymous reviewers for their insightful comments on an earlier draft of this manuscript.

Address correspondence to M. Stroebe or H. Schut, Centre for Bereavement Research \& Intervention, Research Institute for Psychology \& Health, Utrecht University, P.O. Box 80140, 3508 TC, Utrecht, The Netherlands. 
as the above statements illustrate, contemporary researchers differ in their conclusions about the efficacy of working through grief in coming to terms with loss. In recent years, in fact, researchers have begun to question this conceptualization of adaptive coping (e.g., Rosenblatt, 1983; Silver \& Wortman, 1980; Stroebe, 1992; Wortman \& Silver, 1987). It is important to increase our understanding of what comprises effective coping with bereavement, for bereavement is a life event associated with much distress and with physical and mental health detriments (Parkes, 1996; Stroebe, Stroebe, \& Hansson, 1993). There are good reasons to assume that certain coping strategies enable some people to come to terms with loss and avoid severe health consequences, whereas others adopt strategies that are detrimental to health. Thus, better understanding of what comprises adaptive versus maladaptive coping should enable us to predict differential health outcome more accurately, and ultimately to work toward reduction of risk among vulnerable individuals.

The origins of the grief work notion can be traced to the 1917 paper of Sigmund Freud, and his concept of "Trauerarbeit" (grief work). Both Lindemann (1944) and Bowlby (1980) incorporated the concept into their own explanations of the grieving process. It is probably true to say that the most impactful in the field of bereavement today is Bowlby's attachment theory. According to Bowlby, working through grief is important for the purpose of rearranging representations of the lost person and, relatedly, of the self. Although this enabled detachment (labeled reorganization in his more recent work) or the breaking of affectional bonds (Bowlby, 1979), at the same time, it also furthered the continuation of the bond, a relocation of the deceased so that adjustment can gradually be made to the physical absence of this person in ongoing life (see Fraley \& Shaver, in press, for a recent appraisal of Bowlby's ideas about loss and bereavement). The concept of grief work has, then, remained central in theoretical formulations, and it has also continued to have influence in applied fields, being incorporated in principles of a wide variety of counseling and therapy programs (cf. Parkes, 1996; Raphael, Middleton, Martinek, \& Misso, 1993; Stroebe, 1992; Worden, 1991).

How can we address the growing concern among bereavement theorists in recent years about the adequacy of an explanation of 
effective coping in terms of working through grief? We outline the grief work hypothesis (as we shall call it) and summarize reasons for contesting it in the first part of the article. We also consider the application of two more recently developed, influential, general theories from related research areas, for understanding the phenomena of bereavement, and review bereavement-specific approaches that have begun to emerge. Some of these perspectives provide useful guiding principles, which we detail, but nevertheless we argue the need for a coping model that is stressor-specific. Thus, in the second part of the article, we outline our dual process model of coping with bereavement, which extends the explanatory framework to try to overcome limitations of previous formulations. We relate this formulation to other contemporary models in the grief area.

\section{Coping with Bereavement : Evaluation of Contemporary Theories}

\section{The Grief Work Hypothesis}

Following accounts to be found in the scientific literature, grief work can be defined as a "cognitive process of confronting a loss, of going over the events before and at the time of death, of focusing on memories and working toward detachment from the deceased. It requires an active, ongoing, effortful attempt to come to terms with loss. Fundamental to current conceptions is the view that one needs to bring the reality of loss into one's awareness as much as possible and that suppression is a pathological phenomenon" (Stroebe, 1992, pp. 19-20). From this definition, we can derive the "grief work hypothesis," which refers to the notion that one has to confront the experience of bereavement to come to terms with loss and avoid detrimental health consequences (Stroebe, 1992).

It is not surprising that the notion of grief work has had such an influence in both theoretical and applied fields, even to the extent that it has become a "blueprint" for coping. Intuitively, it is easy to accept that one must, for example, "give sorrow words": Grief work seems to be at the essence of grieving, and this common belief is reflected in scientific formulations. To do justice to researchers, it 
must be added that, while they did consider grief work to be fundamental to adaptive grieving, their writing reflects awareness of greater complexity. Grief work was a major theme, becoming a guiding principle of coping for many, but minor themes can be identified alongside (cf. Walter, 1996). For example, the writing of Lindemann $(1944,1979)$ shows recognition of the tendency of the bereaved to pull back from grief:

\begin{abstract}
One of the worst pains there is, is sorrow. Having to suffer this, one gradually masters this pain, and gradually gets away from the inclination that one has not to think about it, to be busy with getting the deceased out of one's mind - forgetting it - putting things away which belonged to him; moving into another place so that one will not be reminded of him... . On the other hand, one may suffer through his mourning, and while one does that, do the essentials of grief work, which now becomes important for all people who are faced with a loss. (1979, p. 234)
\end{abstract}

However, although there has perhaps been some oversimplification, even unfair representation of classic contributions, it is, in our view, fair to say that they considered the grief work hypothesis to be fundamental, that other processes were more implicitly than explicitly documented, and that it has consequently been the grief work hypothesis that has dominated much of our thinking in the bereavement field.

\title{
Shortcomings of the Grief Work Hypothesis
}

As noted above, a number of grief theorists have recognized the limitations of the grief work formulation and questioned the acceptance of it as a dominant principle. In a seminal contribution, Rosenblatt (1983) analyzed 19th-century descriptions of how people dealt with grief, to evaluate contemporary scientific research on coping, and in particular, to assess the validity of the grief work notion: Although the emotional experience was found to be similar in the two centuries, people in the 19th century did not struggle to detach themselves, their memories and hopes, from the deceased. In an influential paper, based on their analyses of some empirical work, Silver and Wortman (1980) argued the case that working through was not only not associated with recovery but even detrimental to it. In a monograph that has helped to redefine 
principles of grief intervention, Worden (1991) reformulated the process of grieving in terms of distinct tasks that the bereaved have to undertake.

In a review of theoretical and empirical research on grief work, following some of the leads of the earlier theorists, Stroebe (1992) summarized a number of shortcomings associated with the grief work hypothesis. The main points of criticism concerned the lack of clarity in the definition of grief work (e.g., the confounding of negative associated rumination with more positively associated aspects of working through), the poor quality of operationalizations in empirical studies (e.g., grief work operationalized as yearning and pining), the absence of sound evidence for it (some studies failed to confirm that confrontation of grief is a predictor of adaptation) and the lack of apparent application across cultures (prescriptions other than the grief work hypothesis exist: These appear to be associated too with adaptation). These criticisms still pertain today.

However, whereas the earlier conclusion argued for more empirical testing and refinement of conceptualization, there are now good reasons to argue, in addition, for a revised model of coping. In our view, there are two main reasons for this, namely, limitations in scientific representation of bereavement phenomena within the grief work framework and its lack of general application.

\section{Inadequate Representation of Bereavement-Related Phenomena}

The first concern, whether the observed phenomena of grieving are adequately represented in the grief work hypothesis, can be illustrated by looking at (a) the definition of the stressor bereavement, (b) process, and (c) outcome variables.

Bereavement as stressor: The lack of specification. Lacking in grief work formulations in general is specification of precisely what has been lost and what has changed through bereavement. There has been a lack of recognition of the range of stressors, the multiplicity of losses, integral to the bereavement experience. Not only is there the loss of the person, but adjustments have to be made with respect to many aspects of life (cf. Worden, 1991). Such secondary stressors also need to be dealt with and (re)appraised, just as the 
meaning associated with the death of the valued person per se needs repeatedly to be thought through, even "pained through" (Lindemann, 1979). As Neimeyer (1998) noted, adaptation to loss involves the restoration of coherence to the narrative of our lives. We return to consider the two types of stressor later on.

\section{Process Variables: A Non-dynamic, Intrapersonal Conceptualization}

The dynamics of confrontation-avoidance. With respect to process, the grief work hypothesis conveys a sense for the need to confront the pain and work through the loss. This is understood to be an effortful process. Yet, the dynamic process that reflects the realization of loss, on the one hand, and the fight against the reality of loss, on the other hand, is neglected in this conceptualization. Denial too takes place at times, it is likewise effortful and (particularly in earlier formulations) it is claimed to be detrimental to health. Representation of the tendency, even necessity (as we will argue later) to confront combined with the tendency to avoid, deny, or suppress aspects of grieving as part of the coping process is still needed in scientific analysis. We need to examine further the extent to which confrontation and avoidance of primary and secondary stressors takes place and is efficacious.

Coping in sociallinterpersonal context. This dynamic process of coming to terms with a death does not take place in isolation. The bereaved are surrounded by others, some of whom are, likewise, grieving for the deceased. Implicit in the grief work model is the notion that one can work through grief with the help of others. However, the focus has been essentially intrapersonal: When the social setting of grief is considered (even this is rare) it is still the impact on individual adjustment that is the focus of analysis. Neglected so far (though of potential integration also in existing formulations) is analysis at an interpersonal level. Interactions with others can be expected to affect the grieving process and adjustment on both intra- and interpersonal levels. For example, discordance in the expression of distress between a bereaved mother and father can be interpreted in terms of more or less grieving by each partner, and affect their relationship, well-being, and the way they cope together and apart. 
Outcome variables: "Medical model'" focus. With respect to outcome variables, the grief work formulation leads one to focus on the potential link between a confrontational style of grieving and outcome in terms of psychological and physical symptomatology. Adherence to a grief work framework limits the focus largely to health outcome factors, even though, of course, well-being and adjustment are of central interest. Nevertheless, there has been a tendency to neglect other "products" of grieving (cf. Walter, 1996), for example, "positive growth" outcomes (Tedeschi \& Calhoun, 1995), creation of a durable narrative about the deceased, reconstruction of the meaning of the deceased in ongoing life (Walter, 1997), and emergence of different roles and identities. Positive outcomes in addition to individual health indicators include relationship maintenance and restored functioning of the family as a unit (e.g., reassignment of the roles of the deceased to other members). The alternative, narrow view of functioning runs the risk that grief will be understood in terms of psychological and medical problems: Something that needs curing, needs to be overcome, is bad. There is the implicit assumption that, after the death of a close person, one must return to a positive state of mind and well-being as soon as possible. The danger, following this line of reasoning, is that human suffering, integral to grief as we know it, will be considered bad, and that the human condition should only, ideally, encompass positive states and emotions, a view that is far from universal (cf. Buddhist beliefs).

\section{Lack of Universal Application}

The grief work hypothesis has been suggested as a general explanation of functional coping. Yet, its relevance to understanding and explanation of gender and cultural patterns is questionable.

Gender specificity. First, the grief work hypothesis does not take adequate account of preferred masculine ways of going about grieving, which are typically less confrontive with respect to the emotion of grief, and less overtly expressive of distress and depression than those found among females (Stroebe, 1998; Stroebe, Stroebe, \& Schut, unpublished manuscript). Although male grieving has recently received some scientific attention (e.g., Lund, in press), in the past female grief has been much more studied. As 
Carverhill and Chartier (1996) have described, this is "A reversal of the usual trend in psychological research to generalize from a largely male sample" (p. 1). The question arises, then, whether the grief work hypothesis that has been derived from the study of a largely female sample is, in fact, generalizable to a male sample: Is what we have at present a "female model of grieving"? The answer to this question is more complex than might appear. It will be discussed below, in the light of empirical evidence.

Cultural specificity. Second, we contend that the grief work hypothesis is culture-bound, at least with respect to the overt level of grief. Different conceptualizations of acceptable or "healthy" ways of coping are to be found in non-Western cultures (Stroebe \& Schut, 1998; Stroebe \& Stroebe, 1987). Some cultures show little or no evidence of "working through" patterns, for apparently this would be considered detrimental to the health of the bereaved and those around them (e.g., among the Muslim community of Bali, according to Wikan, 1990). In other cultures, the bereaved appear to work through normality in very different ways from western understanding of "normality," for example, mutilating the body, or tearing of the hair, as evidenced in some Aboriginal tribal peoples (see Stroebe \& Stroebe, 1987).

\section{General Theories of Coping with Stress}

Looking beyond the bereavement literature to research on stressful life events and coping with trauma in general, two theoretical perspectives contribute potentially useful paradigms, namely, cognitive stress theory (Folkman \& Lazarus, 1980; Lazarus \& Folkman, 1984) and Horowitz's (1986) analysis in terms of stress response syndromes. Because they have considerable bearing on the formulation of our stressor-specific model, and because general stress theories should prove valuable for predicting reactions to specific stressors such as bereavement, the relevant parameters of these theories are outlined next.

\section{Cognitive Stress Theory}

Following cognitive stress theory, stress is experienced by an individual when the demands of a given situation are seen as 
taxing or exceeding resources, which endangers well-being and health. Cognitive appraisal processes operate to determine whether a situation should be categorized as challenging or stressful (cf. Lazarus \& Folkman, 1984). Major dimensions of the coping process to master stressful situations are problem- and emotionfocused coping, the former being directed toward managing and altering the problem that is causing the distress, the latter toward the management of the resulting emotion (see Billings \& Moos, 1981). The former are said to be more appropriate in situations that are changeable, the latter in unchangeable situations.

Cognitive stress theory offers a much finer-grained analysis for understanding the process of coping with bereavement than the grief work formulation. In fact, the critical analysis offered with respect to the latter model in the preceding section of this article can be seen to follow the component analysis of cognitive stress theory, that is, in terms of characteristics of the stressor, the coping process, and the outcome. So, does cognitive stress theory provide an alternative framework? While it has much to add to our understanding on a general level, further specification with respect to all three dimensions seems necessary.

Within this framework, the global stressor is bereavement. As noted above, bereavement entails a number of specific stressors. The major one of these, as can be reasoned from attachment theory, is the primary loss of the attachment figure. Other simultaneous and likewise ongoing stressors, frequently mentioned by the conjugally bereaved, are such factors as financial and skills losses that had been contributed by the deceased (cf. Gentry, Kennedy, Paul, \& Hill, 1995; Worden, 1996). While an advantage of this framework is its detailing of primary and secondary stressors, it would seem reasonable to argue that these stressors are all of importance. The model incorporates the possibility that different stressors co-exist but does not describe a process of concurrent appraisal and coping with different stressors, but rather appraisal and coping with one specific stressor (cf. Folkman et al., 1991, Figure 1).

Some aspects of bereavement are changeable, others are not. One cannot bring back the loved one, but one may be able to change the financial situation for the better by going out to work. So, should the former, as the theory proposes, be dealt with in an 
emotion-focused way, the latter in a problem-focused way? Predictions about outcome already become difficult to make. Furthermore, it is evident that emotion-focused coping incorporates both the control and the expressivity of grieving, which cuts across such diverse categories as rumination, working through versus avoidance, suppression, or denial. One needs to differentiate precisely between adaptive and maladaptive emotion-focused coping methods, but this may be difficult to do and it certainly makes predictions even more difficult (cf. Billings \& Moos, 1984).

A further dimension of coping has featured prominently in cognitive stress theory research, namely, confrontation-avoidance (cf. de Ridder, 1997). This dimension is also problematic in the bereavement context. For example, one may be avoidant in dealing with the emotion of grief, but, at the same time, confrontive of emotions to do with the other stressors identified above.

In conclusion, it becomes evident that there are difficulties in applying the model to bereavement. Bereavement is a life event incorporating multiple stressors, some of which may be appraised as changeable, others not. Problem-focused coping would seem appropriate for changeable aspects, emotion-focused for unchangeable. Thus, successful outcome would necessitate both. Both then again, looking closer, the distinction between emotion- and problem-focused coping itself seems unclear in the context of bereavement. Bereavement is a life stressor eliciting grief, an emotion. What needs to be coped with above all is grief. An emotion itself becomes the stressor, perhaps to a greater extent than in the case of other stressors (e.g., rape, burglary) because it is hard to control its overt expression, and lack of control of the emotion of grief presents difficulties for the self and others. How, then, does one deal with this distressing emotion in an emotionversus problem-focused way, given that emotion-focused coping incorporates the control of emotions as well as the expression of them? Apparent problem-focused behavior may, indeed, be emotion-focused.

\section{Horowitz's Stress Response Syndrome}

Given the overlap between the experiences of trauma and bereavement, it stands to reason that theorizing in the former more 
general area should be relevant to analysis of phenomena in the latter. The work of Horowitz (e.g., 1986) offers most potential in this respect.

The stress response "syndrome," as Horowitz terms it, describes normal manifestations following the experience of a traumatic event (i.e., the personal experience of drastic, horrendous, unpleasant, shocking events). The difference between normal and disordered reactions to trauma is said to lie in the intensity and frequency, rather than the type of reactions that occur (van den Bout, Kleber, \& Brom, 1991; Kleber \& Brom, 1992). Horowitz (1986) identified antithetical reactions of intrusion and avoidance, which would seem to be the most distinctive feature of trauma reactions. Intrusion is the compulsive re-experiencing of feelings and ideas to do with the event, including sleep and dream disturbance and hypervigilance. Avoidance signifies a denial process, and includes reactions such as amnesia, inability to visualize memories, and evidence of disavowal. These extreme deflections between "too much" and "too little" conscious experience may either be simultaneous manifestations, or a sequence of phases may be evident, a conception that, though not always occurring, is basic to Horowitz's model (cf. Horowitz, Bonanno, \& Holen, 1993).

Horowitz's (1986) concern in describing intrusion-avoidance processes was to define the extremity of the reaction to a trauma. The latter processes were symptomatic, useful for classification in the diagnosis of pathology, extreme intrusion-avoidance describing disturbance. By contrast, our concern is to come to an understanding of differences between effective versus ineffective coping processes as mediators in the reduction of symptomatology as outcome variables. Thus, there is a subtle but fundamental difference in the nature of the scientific question in the two cases, with Horowitz asking "How impactful was the event (in terms of intrusionavoidance)?" whereas we would ask "Are (confrontationavoidant) coping strategies leading to adjustment to the event?" Put simply, Horowitz seeks to understand the causes of (extreme) intrusion-avoidance, we seek to understand the effects of processes of confrontation and avoidance. Thus, while intrusion-avoidance may help us to represent a dynamic coping process, we will be using the dimension differently from Horowitz's formulation. 
The intrusion-avoidance process that Horowitz (1986; Horowitz, Stinson, \& Field, 1991) has described is not simply in terms of a voluntary process, but also, critically, an involuntary (lack of personal control over) focus of cognitions. This cannot be assumed for bereavement. Although the type of processing under investigation in the case of general trauma and more limited bereavement may be similar with respect to voluntary and involuntary control processes, we suggest that there is a difference in the balance, that trauma reactions would tend to incorporate comparatively more involuntary, bereavement more voluntary processing. This needs further investigation in future research.

In our view, differences with respect to content, rather than the structural ones discussed above, are more striking. There are differences in the nature of the experience under investigation so far. Study of the aftermath of trauma has focused primarily on the impact of the traumatic event itself, whereas bereavement research has focused on pre- and post-bereavement experience as well as the death event itself. This difference is reflected in dimensions selected to operationalize the impact of traumas versus bereavements. The Impact of Event Scale (see Horowitz, Wilner, \& Alvarez, 1979; Horowitz, Wilner, Kaltreider, \& Alvarez, 1980), designed to measure intrusion-avoidance, refers to "it" - the traumatic eventas the impact indicator. In bereavement, "it" as an impact indicator has had to include other aspects than those relating to the death event itself. As argued above, bereavement incorporates confrontation-avoidance of many different things: thinking about the deceased, events leading up to the death, and the death event itself, as well as the implications for ongoing, altered life (coping with changed circumstances and identity). The latter requires a more extended framework than is provided in the stress response syndrome formulation.

\section{Specific Theories of Coping with Bereavement}

As noted above, a number of alternative, bereavement-specific models have recently been suggested (e.g. Cook \& Oltjenbruns, 1998; Neimeyer, 1998; Parkes, 1993; Rubin, 1981; Sanders, 1989; Walter, 1996; Worden, 1991). On closer inspection, many of these models focus on specific aspects of the human reaction to loss 
that are different from, though not irrelevant to, the focus on effective ways of coping with bereavement, that is of interest in this article. Thus, some theorists provide detailed description of the stressor(s) and psychological and/or other reactions associated with bereavement (e.g., Parkes, 1993). Others concentrate on identifying predictors of outcome (e.g., Sanders, 1989), or are helpful in understanding and explaining aspects such as meaning (re) construction (e.g., Neimeyer, 1998; Walter, 1996). Another angle that is useful is analysis of the content (tasks) involved in coping with loss (e.g., Worden, 1991). We return to this aspect shortly.

\section{Rubin's Trwo-Track Model of Bereavement}

Relevant for current concerns is the model of Rubin (e.g., 1981, 1993; Rubin \& Schechter, 1997). Rubin's (1981) two-track model of bereavement addressed the bereavement process and its outcome, particularly following child loss. Track I is an outcome track: It focuses on the biopsychosocial reactions to bereavement. Track II describes ways of transforming the bereaved's attachment to the deceased and establishing new forms of ongoing relationship to that person. Thus, the first track identifies consequences incumbent on experiencing a major traumatic stressor, the second describes the attack on the relational bond (cf. Rubin, 1993). These two related but not identical dimensions of loss, functioning, and relationship comprise a dual axis paradigm. Thus, the parents' intense preoccupation with the deceased child "sets in motion" the bereavement response (Rubin, 1993).

It would seem from this formulation that there is compatibility with the more general models described above. Similar dimensions to the two tracks are identifiable among the stressors, appraisal processes, and outcomes by Bowlby (1980), Folkman et al. (1991), and Horowitz (1986). For example, one could say that the stressor (loss of the attachment figure) brought about the health outcome (biopsychosocial reactions). The identification of dual dimensions placed on two axes, the one dimension setting the other in motion, can be composed in Folkman et al.'s model with the process whereby the appraisal is repeated, following unresolved coping attempts. But Rubin's model does not focus on the coping process itself, in the sense that it does not provide an analysis of cognitive 
structures or processes (as Folkman et al., 1991, for example, do) that may mediate between the stressor and its outcomes. The model is important for its identification and clear distinction of the two dimensions consequent to the specific stressor of bereavement.

\section{Cook and Oltjenbrun's Model of Incremental Grief}

Cook and Oltjenbruns (1989/1998), in their Model of Incremental Grief, explained how one loss often triggers another loss, resulting in a magnification of grief with each added loss. Taking a family perspective, they described the "dissynchrony of grief" among bereaved persons grieving together over the loss of a loved one, and the discrepant coping styles used by these different persons. Because of such asymmetry and incongruence there occurs what they call secondary loss, which implies a change in the predeath relationship that is regarded as stressful by the survivors. Emanating from the stressful change in the relationship, some persons experience secondary grief. This is not, then, directly due to the death itself. Cook and Oltjenbruns hypothesized that such secondary grief may contribute to marital discord (in the case of child loss). Grief resulting from primary loss (of a child) may trigger a secondary loss (change in relationship with partner) and precipitate yet another, tertiary loss (e.g., divorce). A shift in focus of grief and experience of multiple losses occurs. This is what these researchers term incremental grief, that is, "the additive factor of grief due to multiple related losses" (p. 160).

This model provides a framework from which hypotheses can be derived and predictions made with respect to outcomes following bereavement. It provides an invaluable family perspective, extending consideration beyond the more intrapersonal dimensions provided in the general theories placing, as it does, individual reactions within the context of the social environment where they take place. This systematization of interpersonal processes complements, and is compatible with, the analysis of cognitive processes that we offer below.

\section{Conclusion}

For the most part, the models described above offer complementary rather than conflicting ideas about coping. The grief work 
formulation captures the essence of grieving over the loss of a loved one per se: we indeed need to confront the reality of death to adjust to life without the person - at least in our culture. But it neglects the need to attend to other stressors than the lost relationship itself, it does not take account of fluctuation of attention in the coping process, and it does not provide a detailed analysis of the dynamic coping process. Many of these shortcomings are overcome in one or other of the general models. Cognitive stress theory suggests ways that we can itemize the range of stressors, examine appraisal processes and ways of coping. Limitations here were identified too though: The model does not reflect the dynamic interplay of approach and avoidance between the various stressors associated with bereavement. Horowitz's stress response syndrome analysis does describe a dynamic process of intrusion-avoidance, but again, we noted problems in using this formulation, which focuses on the extremity of control processes to evaluate the extent of the impact of the traumatic event, as a description of coping efficacy in bereavement (it is significant that complicated traumatic reactions are said to need independent, prior intervention, before grief can take its natural course, see Raphael et al., 1993. This supports the view that the phenomena involved in traumatic and grief reactions are different).

The limitations of the general theories, when applied to the bereavement area, and the concentration of bereavement-specific models on aspects other than cognitive processing, led to the formulation of a stressor-specific model of coping with bereavement. In this model, we have incorporated and adapted ideas from these existing perspectives but added a cognitive process analysis that tries to represent the dynamics of coping with bereavement.

\section{Description of The Dual Process Model}

The Dual Process Model of Coping with Bereavement is a taxonomy to describe ways that people come to terms with the loss of a close person. Originally developed to understand coping with the death of a partner, it is potentially applicable to other types of bereavement. Its application to other loss experiences or traumatic events has not yet been systematically explored. In the following 
description, components of the model are detailed. These include the stressors associated with bereavement, the cognitive strategies involved in coming to terms with this life event, and the dynamic process of oscillation, a component that distinguishes this formulation clearly from previous ones.

\section{Loss- Versus Restoration-Orientation}

We argued the need to specify stressors associated with bereavement. What, then, is the nature of the experienced life changes and losses? Attachment theory focuses on the nature of the loss relationship, cognitive stress theory includes global and specific stressors in the model but does not detail how different stressors can be coped with concurrently, nor what these would be in the case of bereavement. Bereavement researchers and clinicians have indeed listed various adjustments or psychosocial transitions that need to be made (e.g., Lopata, 1993; Marris, 1974; Parkes, 1993), but with the exception of Worden (1991), who has given brief coverage, these have not been considered in relationship to the coping process.

Examination of the phenomena of bereavement suggests that people undertake, in varying proportions (according to individual and cultural variations), what we call loss- and restorationoriented coping. These refer to two categories of stressor, each of which requires coping efforts during bereavement. It is evident that coping does not occupy all of a bereaved person's time: Coping is embedded in everyday life experience, which involves taking time off from grieving, as when watching an engrossing TV program, reading, talking with friends about some other topic, or sleeping. This model is depicted in Figure 1.

Loss-orientation refers to the concentration on, and dealing with, processing of some aspect of the loss experience itself, most particularly, with respect to the deceased person. The grief work concept of traditional theories falls within this dimension, focusing as it does on the relationship, tie, or bond with the deceased person, and it typically involves rumination about the deceased, about life together as it had been, and the circumstances and events surrounding the death. It also encompasses yearning for the deceased, looking at old photos, imagining how he or she would react, or crying about the death of the loved person. It is evident that a 


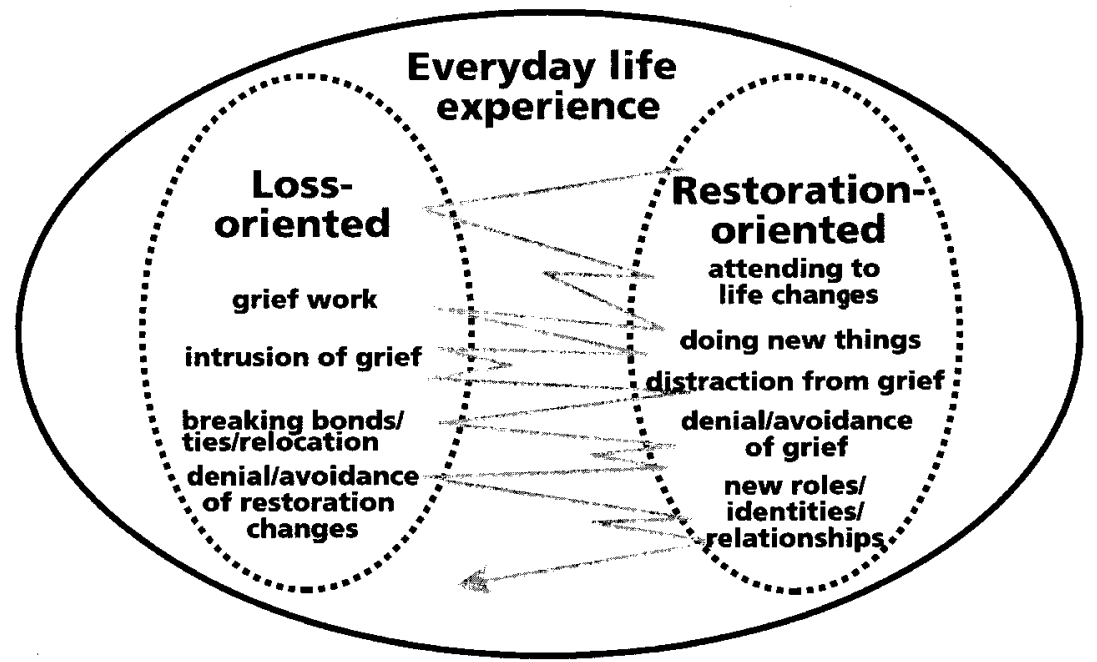

FIGURE 1 A dual process model of coping with bereavement.

range of emotional reactions are involved, from pleasurable reminiscing to painful longing, from happiness that the deceased is no longer suffering to despair that one is left alone.

How does loss orientation relate to the duration of bereavement? Whereas the emotions comes and go, sometimes unwilled and unexpected, in the early days negative affect tends to predominate; however, as time goes on, positive affect plays an increasingly important role in the recovery process. It will already be evident that this model is not a phasal model, we do not propose a sequence of stages, but rather a waxing and wanning, an ongoing flexibility, over time. Early on in bereavement, loss orientation dominates, later on, attention turns more and more to other sources of upheaval and distress.

Loss orientation is not unlike the primary grief dimension postulated by Cook and Oltjenbruns (1998), or the relationship track postulated by Rubin (1981), both of which were described above. All three formulations focus on the nature and closeness of the bond, attachment and continued relationship to the deceased person, identifying such aspects as a major component in grieving, and a major determinant of outcome.

The second type of bereavement stressor is restoration-orientation. With the notable exception of work by Cook and Oltjenbruns 
(1998) on their related (though conceptually narrower) concept of secondary loss, this dimension had not been made as explicit in bereavement research or in counseling programming as has lossorientation. It is important to note that "restoration" does not refer to an outcome variable, but to secondary sources of, and coping with, stress. In other words, this analysis is focusing on what needs to be dealt with (e.g., social loneliness), and how it is dealt with (e.g., by avoiding solitariness), and not with the result of this process (e.g., restored well-being and social reintegration). When a loved one dies, not only is there grief for the deceased person, one also has to adjust to substantial changes that are secondary consequences of loss. In many bereavements these additional sources of stress add considerably to the burden of loss and cause extreme additional anxiety and upset. They include mastering the tasks that the deceased had undertaken (e.g., the finances or cooking); dealing with arrangements for the reorganization of life without the loved one (e.g., it may be necessary to sell one's house); the development of a new identity from "spouse" to "widow(er)" or from "parent" to "parent of a deceased child" (it is noteworthy that there is no single word in our society for this change in identity). Again, a myriad of emotional reactions can be involved in coping with these tasks of restoration, from relief and pride that one has mastered a new skill or taken the courage to go out alone, to anxiety and fear that one will not succeed or despair at the loneliness of being with others and yet on one's own.

The above formulation in terms of restoration orientation is similar to Cook and Oltjenbruns's (1998) secondary loss dimension, although, as noted above, our concept includes other aspects of adjustment than changes in relationship alone. The range and different types of disruptive transitions included under restoration can be illustrated by comparison with Gentry et al.'s (1995) analysis of household consumption patterns following a death. These researchers identified different types of adjustment consequent to different types of loss. Following spousal loss, the biggest adjustment had to be made with respect to household roles (cf. Figure 1), the surviving spouse having to adjust to "new acquisition, maintenance, and disposal responsibilities, at the same time when his/her motivation and ability to adjust are minimal" ( $p$. 77). The death of a child, on the other hand, did not change 
household role structures, but instead affected household communication patterns. Men and women handled their grief very differently, which, they concluded, resulted in redirection of negative emotions toward the spouse. Escalation of problems due to miscommunication or lack of communication could then result (cf. also Cook \& Oltjenbruns, 1989/1998).

The above description is also compatible with the "task model" formulation of Worden (1991) but specifies additional tasks to incorporate the restoration-oriented dimension in the following way. The first task Worden identified was to accept the reality of the loss. In our framework, it is also necessary to accept the reality of the changed world. The second task was to experience the pain of grief. We would argue the additional necessity to take time off from the pain of grief. The third task was to adjust to an environment in which the deceased is missing, referring to external (secondary stressors) as well as internal adjustments (self-assessments) and spiritual adjustments (how one views the universe, etc.). We would add the need to reconstrue the (subjective) environment itself. The fourth task was to relocate the deceased emotionally and move on with life, to which we would add the specification that bereaved persons need to develop new roles, identities, and relationships.

Finally, loss- and restoration-orientation as constructs bear comparison with Parkes's (1993) psychosocial transitions, in which the balance of losses through the death, and gains in terms of positive (ultimate) changes is described. Missing from this latter and other models is analysis of the cognitive processes regulating attention to these different aspects. How we have attempted to build this into our own model is described next.

\section{Oscillation}

This, then, leads to a central component of the model that distinguishes it from classic stress-coping theory or the bereavement models mentioned above, namely, the dynamic process, postulated as fundamental to successful coping, called oscillation. This refers to the alternation between loss- and restoration-oriented coping, the process of juxtaposition of confrontation and avoidance of different stressors associated with bereavement. At times the bereaved will be confronted by their loss, at other times they will avoid 
memories, be distracted, or seek relief by concentration on other things. Sometimes there may simply be no alternative but to attend to the additional stressors (e.g., managing household chores, or earning a living). As such, this cognitive process is a regulatory mechanism that differs too from the "confrontation" versus "avoidant" coping strategies to be found in the general coping research literature (cf. de Ridder, 1997). Our formulation is a dynamic, back-and-forth process. Coping theorists, by contrast, operationalize confrontation versus avoidance as a way of coping reflecting a state or a trait.

Important to stress is the relationship between oscillation and mental and physical health outcome. It is postulated that oscillation is necessary for optimal adjustment over time. A number of reasons why this alternation should take place can be suggested. The person may choose to take "time off," be distracted, or need to attend to new things, or at times it may be too painful to confront some aspect, leading to voluntary suppression or more involuntary repression. In contrast to classic psychoanalytic formulation, which emphasized the detrimental effects, the benefits of denial are acknowledged. This is provided that denial is not extreme and/or persistent. There is supportive evidence that it may be impossible to avoid grieving unremittingly without severe costs to mental and physical well-being, from which it would follow that oscillation is necessary. Suppression of negative emotions is effortful, leading to continued physiological reactivity (e.g., blood pressure and heart rate), and in addition, adverse health effects have been shown, for example, to follow the inhibition of disclosure of trauma (cf. Horowitz, 1986; Pennebaker, 1989, Rimé, Finkenauer, Luminet, Zech, $\&$ Philippot, 1998). It is beyond the scope of this article to review the benefits versus detrimental effects of confrontation-avoidance (for further consideration, see Stroebe and Schut, in press).

In the course of time, after sufficient, perhaps repeated exposure and confrontation, there may no longer be a need to think about certain aspect of the loss. Habituation has taken place. There is also likelihood that reactions become weakened over time, if left inactivated (Kruglanski, 1993). The lines of Emily Bronte come to mind in this context: Have I forgot, my Only Love, to love thee, Severed at last by Time's all-wearing wave? (Bronte in Stallworthy, 1973). 


\section{Implications of the Model}

In our view, the principles outlined above further the understanding of the grieving process. In this final section we illustrate, drawing on the available empirical evidence, how the model may enhance scientific understanding with respect to three key issues; the classification of pathological grief, the analysis of subgroup (gender) differences, and the analysis of the interpersonal and cultural context of bereavement.

\section{Complicated Grief}

The model provides a framework for understanding pathological, or complicated, forms of grief, such as "chronic," "absent," or "inhibited" grief (cf. Lindemann, 1944; Parkes, 1996; Parkes \& Weiss, 1983) as we have come to know them. In the recent formulation of Prigerson and her colleagues (e.g., Prigerson, Frank, et al., 1995; Prigerson, Maciejewski, et al., 1995), the identification of a distinct category of "complicated grief" can be signified as a "loss orientation" syndrome: Items on the Complicated Grief Scale relate for the most part to the lost relationship and continuing attachment to the deceased. On the other hand, grief that is epitomized by denial or inhibition would be more restoration oriented: An avoidance of confrontation with the reality of the death would be typified by an effort to carry on as "normal" in the world, to plunge into work and continue as though nothing had happened. Such pathological forms of grieving can be seen as disturbances of oscillation: In these cases there is an absence of the type of confrontation-avoidance processing (oscillation) that is associated with adjustment.

In cases of extreme rumination or extreme denial, regulation of working through has been shown to be effective for severe (Brom, Kleber, \& Defares, 1989; Horowitz, Marmar, Weiss, DeWitt, \& Rosenbaum, 1984), or mildly complicated (Schut, Stroebe, Stroebe, van den Bout, \& de Keijser, 1994) grief. In line with such empirical results, Kavanagh (1990) argued for a broader model of intervention for those who are encountering difficulty in coping with bereavement, which also appears to acknowledge the type of processing described above in the dual process model. He pointed 
out the narrowness of traditional reliance on exposure and habituation to grief cues, arguing that the challenges encountered in bereavement are multifaceted. Intervention may, for some, be counterproductive - avoidance of grief cues, and, perhaps, confrontation with other tasks such as the restoration tasks described above - would be beneficial.

Different from the types of complication described above are those following a traumatic loss, where postraumatic stress disorder may be apparent (cf. Horowitz, 1986). In the framework of the dual process model, this type of pathology could be described as a disturbance of the oscillation process itself. Intrusion of lossoriented components would be less under voluntary control, would occur in the extreme and violently (e.g. nightmares about the death) and when least expected or wanted. Likewise, extreme avoidance - also sometimes involuntary—would juxtapose with intrusion, possibly manifested as restoration-oriented coping. For example, the person may be apparently functioning in daily life as though no loss had occurred.

\section{Gender Differences}

The model, in our view, seems to be descriptive of male and female ways of grieving. Recent evidence has shown bereaved mothers to be more loss oriented than fathers, following the death of their child (Dijkstra, van den Bout, Stroebe, Stroebe, \& Schut, 1997). Given the gender differences in expressiveness of emotions, mentioned above, this is not surprising. Research from the more general field of sex roles suggests that such style differences may be related to differential outcomes. Helgeson (e.g., 1994) identified the poor health consequences of what she labelled unmitigated communion, a trait she claims to be associated with femininity, or unmitigated agency, which she associates with masculinity. Drawing a general parallel between these constructs and loss versus restoration orientation seems reasonable. The implication would then be that either extreme, either female or male, is "unhealthy."

That bereaved men and women may indeed follow their gender specific way, to the detriment of their health, has been suggested in a small study that needs replication. Investigating the expressed wishes about what men and women want from family bereavement 
support groups, Hopmeyer and Werk (1994) asked how much time should be devoted to specific aspects. Female respondents gave first place to "Sharing feelings and emotions," while this ranked tenth among male respondents, who ranked "Learning how others solve problems like mine" as first.

Further, indirect support for the model's applicability to patterns of gender differences comes from an intervention study (Schut, Stroebe, de Keijser, \& van den Bout, 1997). This program offered grief counseling to widows and widowers for mildly disturbed grief. The participants had been bereaved for some time (ranging from 11 to 18 months after their loss). They were randomly assigned to individual intervention that either followed client-centered or behavioral therapy guidelines. Thus, the former was more directed at the open expression of emotion, the latter at dealing with the problems associated with the bereavement situation. Results showed a remarkable sex by intervention condition interaction effect: "Teaching" bereaved men and women to cope in the way that the opposite sex usually adopts (teaching men to be more emotion-oriented and women to be more problem-oriented) was associated with a lowering of distress. Focusing on traditional sex-role coping patterns hardly had any effect.

\section{The Social and Cultural Context of Grieving}

In contrast to the more intrapersonal models that have been developed in the past, the model is appropriate for examining interpersonal in addition to intrapersonal processes in adjustment to bereavement, and for the understanding of grieving in social context, where the way of grieving of one person will impact and change that of another (cf. Dijkstra et al., 1997). The gender differences example illustrates this. If a mother's grief over the loss of a child is more loss-oriented, a father's more restoration-oriented, this discordance in ways of coping may, at times, be mutually unconducive. It would be easy for a mother to make the attribution that "He is grieving less than I am" rather than, what might be the case that "He is grieving differently from the way I am."

Similarly, the model can account for cultural differences (see Stroebe \& Schut, 1998). Although grief is essentially a universal human reaction to loss of a significant other, cultural prescriptions 
impact on the way that grief is manifested. It is useful to describe cultural differences along the loss-restoration dimension. For example, as Wikan (1988) has described, the Muslim community on the island of Bali would be described as restoration-oriented: There is little expression of grief, and crying is not sanctioned, despite the fact that a death is felt keenly. On the other hand, the Muslim community in Egypt expresses their grief openly, gathering to reminisce and share their anguish over loss. Underlying both patterns are cultural belief systems that dictate the way that grief is manifested and expressed.

\section{Conclusion}

Confrontation with the reality of loss is the essence of adaptive grieving. It needs to be done, the cognitive business needs to be undertaken, but not relentlessly, and not at the expense of attending to other tasks that are concomitant with loss. It needs "dosage." This is the most central claim of the dual process model that distinguishes it from other formulations. However, until further research has been done, caution must be observed in making assumptions, or in deriving practical implications from assumed regularities. The task now is to provide further empirical verification for the components of the dual process model of coping with bereavement, for example, the process itself of oscillation and its efficacy. What needs to be examined in addition to the more structural components is the content of cognitive activity in coping with loss: What positive and negative meanings, reappraisals, and attributions underlie the ability to regulate grieving and make progress through one's grief? We are currently working at this level of analysis, to provide understanding of cognitive processing within the dual process model outlined above.

\section{References}

Billings, A. G., \& Moos, R. H. (1981). The role of coping responses and social resources in attenuating the stress of life events. Fournal of Behavioral Medicine, 4, $139-157$. 
Billings, A. G., \& Moos, R. H. (1984). Coping, stress, and social resources among adults with unipolar depression. Journal of Personality and Social Psychology, 46, 877-891.

Bowlby, J. (1979). The making and breaking of affectional bonds. London: Tavistock.

Bowlby, J. (1980). Attachment and loss. Vol. 3. Loss: Sadness and depression. London: Hogarth.

Brom, D., Kleber, R. J., \& Defares, P. B. (1989). Brief psychotherapy for posttraumatic stress disorders. Fournal of Consulting and Clinical Psychology, 57, 607612.

Brontë, E. (1973). Remembrance. In J. Stallworthy (Ed.). The Penguin book of love poetry. Hammondsworth, England: Penguin.

Carverhill, P. A., \& Chartier, B. M. (1996). A phenomenology of masculine grief. Paper presented at the XXVI International Congress of Psychology, Montreal, Canada.

Cook, A. S. \& Oltjenbruns, K. A. (1998). The bereaved family. In A. S. Cook \& K. A. Oltjenbruns (Eds.), Dying and grieving: Life span and family perspectives (pp. 91-115). Fort Worth, TX: Harcourt Brace.

de Ridder, D. (1997). What is wrong with coping assessment? A review of conceptual and methological issues. Psychology and Health, 12, 417-431.

Dijkstra, I., van den Bout, J., Stroebe, M., Schut, H., \& Stroebe, W. (in press). Coping with the death of a child. A longitudinal study of discordance in couples. Gedrag \& Gezondheid.

Folkman, S., Chesney, M., McKusick, L., Ironson, G., Johnson, D., \& Coates, T. (1991). Translating coping theory into an intervention. In J. Eckenrode (Ed.), The social context of coping (pp. 239-260). New York: Plenum.

Folkman, S., \& Lazarus, R. S. (1980). An analysis of coping in a middle-aged community sample. Journal of Health and Social Behavior, 21, 219-239.

Fraley, R. C., \& Shaver, P. R. (in press). Loss and bereavement: Attachment theory and recent controversies concerning "grief work" and the nature of detachment. In J. Cassidy \& P. R. Shaver (Eds.), Handbook of attachment theory and research. New York: Guilford.

Freud, S. (1984). Mourning and melancholia. In A. Richards (Ed.). The Pelican Freud library volume II (pp. 251-268). Hammondsworth, U.K.: Penguin Books.

Gentry, J., Kennedy, P., Paul, G., \& Hill, R. (1995). Family transition during grief: Discontinuities in household consumption patterns. Fournal of Business Research, 34, 67-79.

Helgeson, V. S. (1994). Relation of agency and communion to well-being: Evidence and potential explanations. Psychological Bulletin, 116, 412-428.

Hopmeyer, E., \& Werk, A. (1994). A comparative study of family bereavement groups. Death Studies, 18, 243-256.

Horowitz, M. (1986). Stress response syndromes. Northvale, NJ : Aronson.

Horowitz, M., Bonanno, G., \& Holen, A. (1993). Pathological grief: Diagnosis and explanation. Psychosomatic Medicine. 55, 260-273.

Horowitz, M., Marmar, C., Weiss, D. S., DeWitt, K. N., \& Rosenbaum, R. (1984). Brief psychotherapy of bereavement reactions. The relationship of process to outcome. Archives of General Psychiatry, 41, 438-448. 
Horowitz, M., Stinson, C., \& Field, N. (1991). Natural disasters and stress response syndromes. Psychiatric Annals, 21, 556-562.

Horowitz, M., Wilner, N., \& Alvarez, W. (1979). Impact of Event Scale: A study of subjective stress. Psychosomatic Medicine, 41, 209-218.

Horowitz, M., Wilner, N., Kaltreider, N., \& Alvarez, W. (1980). Signs and symptoms of post traumatic stress disorders. Archives of General Psychiatry, 37, 85-92.

Kavanagh, D. G. (1990). Towards a cognitive-behavioral intervention for adult grief reactions. British Fournal of Psychiatry, 157, 373-383.

Kleber, R. \& Brom, D. (1992). Coping with trauma: Theory, prevention and treatment. Amsterdam: Swets \& Zeitlinger.

Kruglanski, A. (1993). Discussant's comments. In A. Baum (Chair), Cognitive processes in traumatic life events. Meeting of the American Psychological Society, Chicago, Illinois.

Lazarus, R., \& Folkman, S. (1984). Stress, appraisal, and coping. New York: Springer.

Lindemann, E. (1944). Symptomatology and management of acute grief. American Journal of Psychiatry, 101, 141-148.

Lindemann, E. (1979). Beyond grief: Studies in crisis intervention. New York: Aronson.

Lopata, H. (1993). The support systems of American urban widows. In M. Stroebe, W. Stroebe, \& R. O. Hansson (Eds.), Handbook of bereavement: Theory, research and intervention (pp. 381-396). New York: Cambridge University Press.

Lund, D. (in press). Men's grief. Amityville, NY. : Baywood.

Malkinson, R. (1996). Cognitive behavioral grief therapy. Fournal of RationalEmotive \& Cognitive-Behavior Therapy, 14, 155-171.

Marris, P. (1974). Loss and change. New York: Pantheon Books.

Neimeyer, R. (1998). The lessons of loss: A guide to coping. New York: McGraw Hill.

Parkes, C. M. (1993). Bereavement as a psychosocial transition: Processes of adaptation to change. In M. Stroebe, W. Stroebe, \& R. O. Hansson (Eds.), Handbook of bereavement: Theory, research and intervention (pp. 91-101). New York: Cambridge University Press.

Parkes, C. M. (1996). Bereavement: Studies of grief in adult life (3rd ed.). London: Routledge.

Parkes, G. M., \& Weiss, R. (1983). Recovery from bereavement. New York: Basic Books.

Pennebaker, J. W. (1989). Confession, inhibition, and disease. Advances in Experimental Social Psychology, 22, 211-244.

Prigerson, H., Frank, E., Kasl, S., Reynolds, C., Anderson, B., Zubenko, G., Houck, P., George, C., \& Kupfer, D. (1995). Complicated grief and bereavement-related depression as distinct disorders: Preliminary empirical validation in elderly bereaved spouses. American fournal of Psychiatry, 2, 1-12.

Prigerson, H., Maciejewski, P., Reynolds, C., Bierhals, A., Newsom, J., Fasiczka, A., Frank, E., Doman, J., \& Miller, M. (1995). The inventory of complicated grief: A scale to measure certain maladaptive symptoms of loss. Psychiatry Research, 59, 65-79. 
Raphael, B. Middleton, W., Martinek, N., \& Misso, V. (1993). Counseling and therapy of the bereaved. In M. Stroebe, W. Stroebe, \& R. O. Hansson (Eds.), Handbook of bereavement: Theory, research, and intervention (pp. 427-453). New York: Cambridge University Press.

Rimé, B., Finkenauer, C., Luminet, O., Zech, E., \& Philippot, P. (1998). Social sharing of emotion: New evidence and new questions. In W. Stroebe \& M. Hewstone (Eds.), European Review of Social Psychology (Vol. 9), Chichester, England: Wiley.

Rosenblatt, P. G. (1983). Bitter, bitter tears: Nineteenth century diarists and twentieth century grief theories. Minneapolis: University of Minnesota Press.

Rubin, S. (1981). A two-track model of bereavement: Theory and application in research. American Fournal of Orthopsychiatry, 51, 101-109.

Rubin, S. (1993). The death of a child is forever: The life course impact of child loss. In M. Stroebe, W. Stroebe, \& R. O. Hansson (Eds.), Handbook of bereavement: Theory, research, and intervention (pp. 285-299). New York: Cambridge University Press.

Rubin, S., \& Schechter, (1997). Exploring the social construction of bereavement: Perceptions of adjustment and recovery in bereaved men. American Fournal of Orthopsychiatry, 67, 279-289.

Sanders, C. M. (1989). Grief: The mourning after. New York: Wiley.

Schut, H. A. W., Stroebe, M., Stroebe, W., van den Bout, J., \& de Keijser, M. A. (1994). Are accepted ways of coping with loss really efficacious? Paper presented at the Fourth International Conference on Grief and Bereavement in Contemporary Society, Stockholm, Sweden.

Schut, H. A. W., Stroebe, M., de Keijser, J., \& van den Bout, J. (1997). Intervention for the bereaved: Gender differences in the efficacy of grief counselling. British Journal of Clinical Psychology, 36, 63-72.

Silver, R., \& Wortman, C. (1980). Coping with undesirable life events. In J. Garber \& M. E. P. Seligman (Eds.), Human helplessness: Theory and applications (pp. 279-340). New York: Academic Press.

Stroebe, M. (1992). Coping with bereavement: A review of the grief work hypothesis. Omega, 26, 19-42.

Stroebe, M. (1998). New directions in bereavement research: Exploration of gender differences. Palliative Medicine, 12, 5-12.

Stroebe, M., \& Schut, H. A. W. (1998). Culture and grief. Bereavement Care, 17, 7-10.

Stroebe, M., \& Schut, H. (in press). Meaning making in the dual process model of coping with bereavement. In R. A. Neimeyer (Ed.), Meaning reconstruction and the experience of loss. Washington: American Psychological Association Press.

Stroebe, M., \& Stroebe, W. (1987). Bereavement and health. The psychological and physical consequences of partner loss. Cambridge, England: Cambridge University Press.

Stroebe, M., Stroebe, W., \& Hansson, R. O. (Eds.). (1993). Handbook of bereavement: Theory, research, and intervention. New York: Cambridge University Press.

Stroebe, M., Stroebe, W., \& Schut, H. A. W. (inpublished manuscript). Sex differ- 
ences in adjustment to bereavement: An empirical and theoretical review (Manuscript under revision).

Tedeschi, R. G., \& Calhoun, L. G. (1995). Trauma and transformation: Growing in the aftermath of suffering. Thousand Oaks, CA: Sage.

van den Bout, J., Kleber, R., \& Brom, D. (1991). Traumaverwerking en rouw: eenheid in verscheidenheid [Coping with trauma and grief: unity in diversity] In J. Winnubst, P. Schnabel, J. van den Bout, \& M. van Son (Eds.), De metamorfose van de klinische psychologie. [The metamorphosis of clinical psychology] (pp. 73-85). Assen: Van Gorcum.

Walter, T. (1996). A new model of grief: Bereavement and biography. Mortality, $1,7-25$.

Walter, T. (1997). Letting go and keeping hold: A reply to Stroebe. Mortality, 2, 263-266.

Wikan, U. (1988). Bereavement and loss in two Muslim communities: Egypt and Bali compared. Social Science and Medicine, 27, 451-460.

Wikan, U. (1990). Managing turbulent hearts: A Balinese formula for living. Chicago: University of Chicago Press.

Worden, J. W. (1991). Grief counseling and grief therapy: A handbook for the mental health practitioner. New York: Springer.

Worden, J. W. (1996). Children and grief: When a parent dies. New York: Guilford Press.

Wortman, C. B., \& Silver, R. C. (1989). The myths of coping with loss. Fournal of Consulting and Clinical Psychology, 57, 349-357. 